\title{
Three Cases of Severe Stercoral Colitis
}

\author{
Kashio Toyoda, Keiichi Yokoyama, Tomohide Matsushima, Naoki Hashimoto, Yasuhide Kitazawa
}

Department of Emergency and Critical Care Medicine, Faculty of Medicine, Kindai University, Osaka, Japan

Email: kitaz@med.kindai.ac.jp

How to cite this paper: Toyoda, K., Yokoyama, K., Matsushima, T., Hashimoto, N. and Kitazawa Y. (2018) Three Cases of Severe Stercoral Colitis. Open Journal of Emergency Medicine, 6, 82-87. https://doi.org/10.4236/ojem.2018.64010

Received: September 19, 2018

Accepted: November 27, 2018

Published: November 30, 2018

Copyright (c) 2018 by author(s) and Scientific Research Publishing Inc. This work is licensed under the Creative Commons Attribution International License (CC BY 4.0).

http://creativecommons.org/licenses/by/4.0/

\begin{abstract}
Stercoral colitis is the inflammation of the colonic wall caused by fecal impaction. When a patient with a history of chronic constipation visits the Emergency Center with diffuse abdominal pain, sepsis and or rectal bleeding, clinicians should consider the possibility of stercoral colorectal perforation. An accurate diagnosis on a computed tomography (CT) scan (for fecal impaction, intra-abdominal feces, intraperitoneal free air, stranding of the peri-colonic fat in the segment, presence of extraluminal bubbles of gas) may facilitate early surgical intervention and improve the patient's prognosis based on our clinical experience dealing with three patients who had stercoral colitis.
\end{abstract}

\section{Keywords}

Stercoral Colitis, CT, and Hartman's Operation

\section{Introduction}

Stercoral colitis is an inflammatory colitis related to increased intraluminal pressure caused by impacted fecal material in the colon. This rare condition, first reported in 1894, has been described primarily in the surgical and gastrointestinal literature [1]. As a result of the fecal impaction, a focal pressure colitis may occur with ulceration resulting in colonic perforation. The most severe complication of Stercoral colitis is colonic perforation and a 35\% mortality rate has been reported [2]. If left untreated, ischemic ulceration and perforation can be ensued. The aim of this report is to show that stercoral colitis may lead to colonic perforation and by accurate diagnosis with computed tomography (CT) scan followed by prompt surgery can improve the prognosis. We report the experience of three clinical cases.

\section{Case Report}

Case 1: A 76-year-old man with chronic constipation was admitted to the Emer- 
gency Centre with a complain of severe abdominal pain and vomiting. Abdominal CT showed fecal impaction and intestinal pneumatosis (Figure 1). An emergency laparotomy was performed. During the surgery, we observed a foul-smelling bloody ascites and found necrotic sigmoid colon. We immediately performed resection of the necrotic colon, Harman's operation (rectal excision without anastomosis) and peritoneal lavage. Moreover, residual fecalomas were removed from the remaining rectum. The patient was discharged from the hospital 4 weeks after surgery.

Case 2: A 76-year-old female with atrial fibrillation and brain embolism was admitted to the Emergency Centre with a complain of severe abdominal pain and bloody stool. CT showed fecal impaction of sigmoid colon and free air in the retroperitoneum (Figure 2). During the surgery, we detected hematoma and perforation of recto-sigmoid junction (Figure 3). We performed the resection of recto-sigmoid junction, Hartman's operation and peritoneal lavage. The patient was discharged from the hospital 2 weeks after surgery.

Case 3: A 87-year-old female was admitted to the Emergency Centre with a complain of vomiting and bloody stool. CT showed massive fecal impaction and intestinal pneumatosis in the sigmoid colon (Figure 4). During the surgery, we observed a foul-smelling ascites and found a necrotic area of $20 \mathrm{~cm}$ length from sigmoid colon to rectum. The necrotic area of $20 \mathrm{~cm}$ was resected. However, the vital signs during the surgery were unstable due to a coagulopathy and metabolic acidosis of $\mathrm{BE}-5.1 \mathrm{mmol} / \mathrm{L}$ (normal range; between -3.0 and $+3.0 \mathrm{mEq} / \mathrm{L}$ ). Therefore, we decided to perform damage control surgery, postponing the completion of surgery. It took 38 hours to obtain stable general clinical conditions at the intensive care unit. Following clinical stabilization, we performed surgery to create the stoma. The patient was discharged from the hospital 4 weeks after surgery.

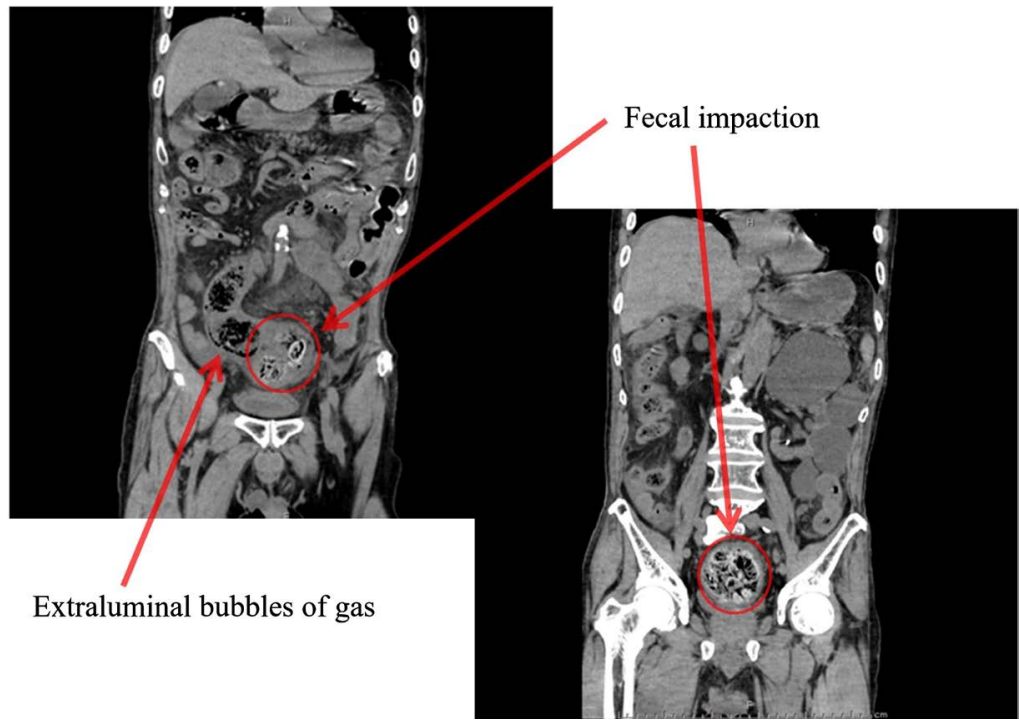

Figure 1. CT scan image of Case 1. The fecal impaction and intestinal pneumatosis in recto-sigmoidal junction were observed. 


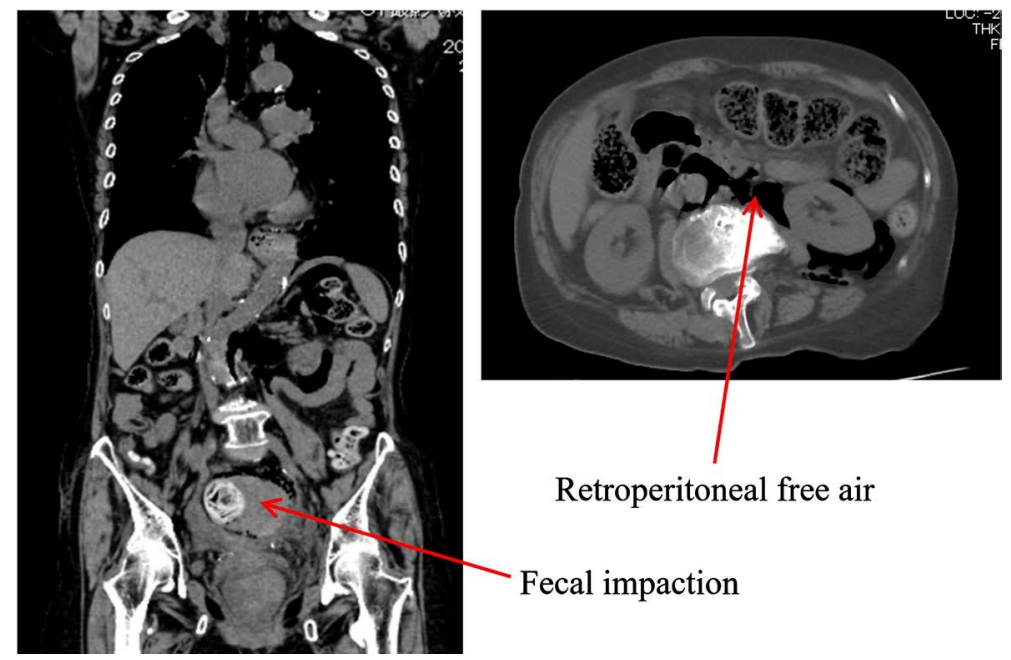

Figure 2. CT scan image of Case 2. The fecal impaction and retroperitoneal free air around the recto-sigmoidal junction were observed.

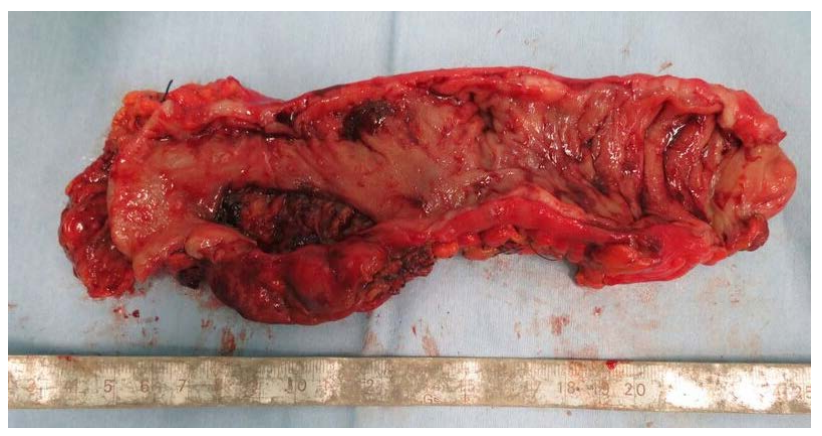

Figure 3. Resected tissue of Case 2. Ovoid perforation of recto-sigmoid colon $(2.5 \times 5$ $\mathrm{cm}$ ) was observed.

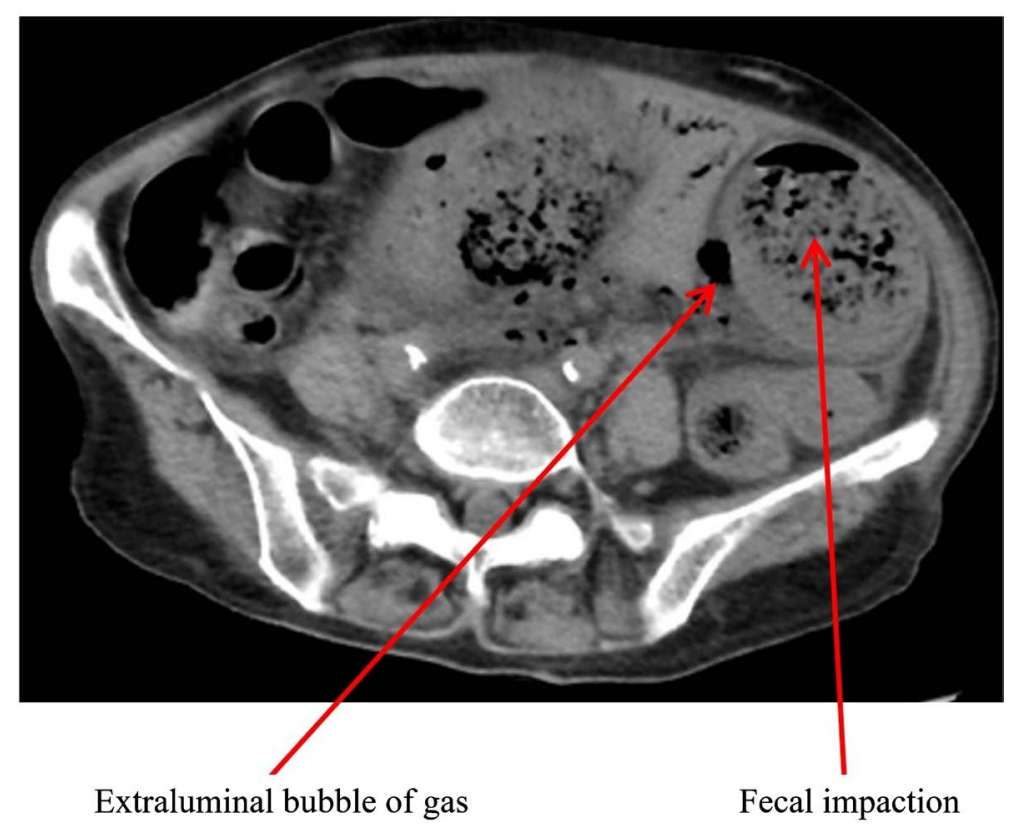

Figure 4. CT scan image of Case 3. Massive fecal impaction and intestinal pneumatosis were observed in the sigmoid colon. 
For each of these three cases, prompt surgery was performed within two hours after diagnosis. All three cases had uneventful postoperative courses and were discharged from the hospital and have been living healthy at home.

\section{Discussion}

Stercoral perforation of the colon is an extremely rare condition. Stercoral perforation of the colon was first reported by Berry [1] to the Pathological Society of London in 1894. According to a recent study, which was one of the largest studies ever carried out by a single institution, the incidence of stercoral perforation of the colon comprised $1.2 \%$ of all cases requiring emergency colorectal surgery and $3.2 \%$ of all those involving colonic perforations [3].

Severe chronic constipation is considered as one of the main causative factors in the development of stercoral perforation of the colon. The fecalomas exert a constant pressure on the bowel wall and lead to pressure necrosis of the mucosa. Several factors help explain why the left hemi-colon is predisposed to stercoral perforation of the colon. Stercoral perforation occurs predominantly in the sigmoid colon/recto-sigmoid junction [2]. This relates to the fact that the stool gets more dehydrated as it passes through the large bowel and that these areas have a somewhat narrower diameter compared with the rest of the large bowel. Equally, stercoral perforation occurs predominantly on the anti-mesenteric border of the colon, and this relates to the fact that these areas have a somewhat meager blood supply.

Huttunen [4] have proposed the following diagnostic criteria for stercoral perforation of the colon. 1) The colonic perforation is round or ovoid, exceeding $1 \mathrm{~cm}$ in diameter, and is orientated anti-mesenterically. 2) Fecalomas are present within the colon, protruding through the perforation site, or lying within the abdominal cavity. 3) Pressure necrosis or ulcer and chronic inflammatory reaction are microscopically present around the perforation site.

Serpell and Nicholls [2] reported that, in 57 patients where the site of perforation was documented, the most common site affected was the sigmoid colon (47\%). The next commonest was the rectosigmoid (30\%) followed by the cecum (9\%). In our cases, all the affected area was located at rectosigmoid colon.

Stercoral perforation is invariably highly symptomatic, with $20 \%$ of patients having clinical features of localized peritonitis, and $80 \%$ of patients having clinical features of generalized peritonitis [2]. CT scans of the abdomen show "diagnostic features" in $90 \%$ of instances (fecal impaction, intra-abdominal feces, intraperitoneal free air, stranding of the peri-colonic fat in the segment, presence of extraluminal bubbles of gas) [5] [6]. The CT images of our patients showed the following symptoms: fecal impaction and extraluminal air in Case 1; fecal impaction of sigmoid colon and retroperitoneal free air in Case 2; and massive fecal impaction and presence of extraluminal bubble of gas in Case 3. Therefore, CT is a very valuable tool for the diagnosis of stercoral colitis and the assessment of its severity.

Mortality of stercoral perforation is reported as high. However, Maurer [3] et 
al provided several factors for a favorable outcome as follows: the patient was 50-year-old or younger, laparotomy was performed within a few hours on admission after diagnosis of perforation in the gastrointestinal tract. The affected colon segment was resected, followed by intraoperatively extensive peritoneal lavage with Ringer's solution, Hartman's operation was the preferred procedure, and no primary anastomosis was performed. Simple closure of the perforated site or segmental resection of the diseased colon with an anastomosis and a diverting enterotomy should be avoided [7].

In Case 1 and 3, CT showed extraluminal gas in the intestinal wall, which suggested intestinal necrosis. In Case 2, CT showed retroperitoneal free air, which suggested intestinal perforation. Two cases had Hartman's operation performed and the third case had damage control surgery due to coagulopathy and severe acidosis. All the cases were successfully discharged from the hospital. CT study is no less worth than physical examinations or laboratory tests for early and accurate diagnosis of severe stercoral colitis. It is very important to search abnormal gas in CT scan images.

\section{Conclusion}

When a patient with a history of chronic constipation visits Emergency Center with diffuse abdominal pain, sepsis and or rectal bleeding, clinicians should consider the possibility of stercoral colorectal perforation. An accurate diagnosis with CT findings may facilitate an early surgical intervention and improve the patient's prognosis.

\section{Conflicts of Interest}

The authors declare no conflicts of interest regarding the publication of this paper.

\section{References}

[1] Berry, J. (1894) Dilatation and Rupture of Sigmoid Flexure (Short Report). British Medical Journal, 1, 301.

[2] Serpell, J.W. and Nicholls, R.J. (1990) Stercoral Perforation of the Colon. British Journal of Surgery, 77, 1325-1329. https://doi.org/10.1002/bjs.1800771204

[3] Maurer, C.A., Renzulli, P., Mazzucchelli, L., Egger, B., Seiler, C.A. and Büchler, M.W. (2000) Use of Accurate Diagnosis Criteria May Increase Incidence of Stercoral Perforation of the Colon. Disease of Colon and Rectum, 43, 991-998. https://doi.org/10.1007/BF02237366

[4] Huttunen, R., Heikkinen, E. and Larmi, T.K. (1975) Stercoraceous and Idiopathic Perforations of the Colon. Surgery, Gynecology \& Obstetrics, 140, 756-760.

[5] Kumar, P., Pearce, O. and Higgindson, A. (2011) Imaging Manifestation of Faecal Impaction and Stercoral Perforation. Clinical Radiology, 66, 83-88. https://doi.org/10.1016/j.crad.2010.08.002

[6] Chakravartty, S., Chang, A. and Nunoo-Mensah, J. (2013) A Systematic Review of Stercoral Perforation. Colorectal Disease, 15, 930-935. 
https://doi.org/10.1111/codi.12123

[7] Kang, J. and Chung, M. (2012) A Stercoral Perforation of the Descending Colon. Journal of the Korean Surgical Society, 82, 125-127.

https://doi.org/10.4174/jkss.2012.82.2.125 